\title{
A Distributed Transmit Antenna Selection Scheme for Fixed-Gain Multi-Antenna AF Relaying Systems
}

\author{
Diana C. González and José Cândido S. Santos Filho \\ School of Electrical and Computer Engineering \\ University of Campinas (UNICAMP) \\ Campinas-SP, Brazil \\ \{dianigon,candido\}@decom.fee.unicamp.br
}

\author{
Daniel B. da Costa \\ Federal University of Ceará (UFC) \\ Sobral-CE, Brazil \\ danielbcosta@ieee.org
}

\begin{abstract}
Antenna selection has arisen as an attractive strategy in multi-antenna systems due to its reduced complexity and cost. In this paper, the outage performance of a distributed transmit antenna selection scheme for dual-hop fixedgain amplify-and-forward relaying systems is investigated. Our analysis considers a multi-antenna source, a multi-antenna relay, and a single-antenna destination, in which selection combining and maximal-ratio combining are employed at the relay and destination receptions, respectively. Lower and upper bounds for the outage probability of the proposed scheme are derived as the sum of two terms, one term given in closed form and the other term given in single integral form. Moreover, asymptotic expressions for these bounds at high signal-to-noise ratio are obtained in closed form. The results show that our scheme presents full diversity order. More importantly, the underlying distributed strategy is shown to perform closely to the costly optimal centralized antenna selection.
\end{abstract}

\section{INTRODUCTION}

Multi-antenna relaying systems are expected to be widely utilized in future wireless communication networks, since the advantages offered by multiple-input multiple-output (MIMO) and relaying techniques can be fully exploited. However, a potential drawback of the use of multiple antennas is their complex hardware and increased cost [1]. To alleviate this, transmit antenna selection (TAS) has arisen as an alternative low-cost low-complexity strategy to capture the advantages of MIMO systems. Nevertheless, in order to provide the knowledge necessary for the transmitter to select the best antenna, the implementation of TAS schemes usually requires feedback channels for acquiring the channel state information (CSI) of all links. The required bits of feedback information vary depending on the number of antennas at the nodes [1]. In this case, when the number of antennas increases, the delay and complexity involved in the selection mechanism may rapidly become prohibitive in practice.

Several works have shown and explored the advantages of TAS schemes, but a substantial amount of CSI feedback has been observed in most of them. For instance, the optimal TAS strategy was studied in [2] for a dual-hop MIMO amplifyand-forward (AF) relaying system, in which an exhaustive search for the best antenna at the source and relay was required. A different optimal TAS strategy was examined in [3] assuming a variable-gain AF relay and Nakagami- $m$ fading. Two suboptimal TAS strategies were also analyzed in [3], in which the feedback overhead was reduced at the expense of system performance. More recently, a distributed TAS scheme was proposed in [4] assuming a single-antenna fixed-gain AF relay.

In this work, we generalize the scheme proposed in [4] by considering a fixed-gain AF relay with multiple antennas in both transmission and reception. Our scheme provides a low and constant delay/feedback overhead, despite of the number of transmit antennas. We derive lower and upper bounds for the outage probability of the proposed scheme as the sum of two terms, one term given in closed form and the other term given in single integral form. Moreover, an asymptotic expression for each of these bounds at high signal-to-noise ratio (SNR) is obtained in closed form. It is noteworthy that such analysis is considerably more intricate than that of the scheme in [4]. Our results show that the proposed scheme presents full diversity order. More importantly, its underlying distributed strategy is shown to perform closely to the costly optimal centralized TAS.

Throughout this paper, $f_{Z}(\cdot)$ denotes the probability density function (PDF) of a generic random variable $Z, E[\cdot]$ denotes expectation, and $\operatorname{Pr}(\cdot)$ denotes probability.

\section{System Model and Antenna Selection Scheme}

\section{A. System Model}

Consider a dual-hop relaying system composed of one source $S$ with $N_{t}$ antennas, one single-antenna destination $D$, and one half-duplex fixed-gain AF relay $R$ with $N_{r r}$ receive antennas and $N_{r t}$ transmit antennas. A time-division multiple access scheme is assumed and all the channels undergo flat Rayleigh fading.

Before data transmission, the transmit antenna at $S$ that maximizes the end-to-end instantaneous SNR is chosen according to the procedure to be described in the next subsection. Afterwards, a conventional cooperative transmission takes place in two time slots. In the first time slot, the signals received by the multiple antennas at $R$ are combined using a selection combining (SC) scheme, and, in the second time slot, the signals coming from the direct and relaying links ${ }^{1}$

\footnotetext{
${ }^{1}$ The relay employs a TAS scheme to forward to the destination the signal received from the source. This will be described next
} 
are combined at $D$ using a maximal-ratio combining (MRC) scheme. The end-to-end SNR from the $i$ th antenna at $S$ to $D$ (through the $k$ th receive antenna and $j$ th transmit antenna at $R$ ) can be written as

$$
\gamma_{i, k, j}=\gamma_{S_{i} D}+\frac{\gamma_{S_{i} R_{k}} \gamma_{R_{j} D}}{\gamma_{R_{j} D}+C}
$$

where $\gamma_{S_{i} D} \triangleq \frac{P_{S}}{N_{0}}\left|h_{S_{i} D}\right|^{2}, \gamma_{S_{i} R_{k}} \triangleq \frac{P_{S}}{N_{0}}\left|h_{S_{i} R_{k}}\right|^{2}, \gamma_{R_{j} D} \triangleq$ $\frac{P_{R}}{N_{0}}\left|h_{R_{j} D}\right|^{2}$, and $C=1+\bar{\gamma}_{S_{i} R_{k}}$, with $\bar{\gamma}_{S_{i} R_{k}}=E\left[\gamma_{S_{i} R_{k}}\right]$. In these expressions, $\left|h_{S_{i} D}\right|^{2},\left|h_{S_{i} R_{k}}\right|^{2}$, and $\left|h_{R_{j} D}\right|^{2}$ denote the channel power gains of the links from the $i$ th antenna at $S$ to $D$, from the $i$ th antenna at $S$ to the $k$ th antenna at $R$, and from the $j$ th antenna at $R$ to $D$, respectively; $P_{S}$ and $P_{R}$ stand for the transmit powers at $S$ and $R$, respectively; and $N_{0}$ denotes the additive white Gaussian noise (AWGN) at each receiving terminal. We assume a homogeneous network, in which $E\left[\gamma_{S_{i} R_{k}}\right]=\bar{\gamma}_{S R}, E\left[\gamma_{S_{i} D}\right]=\bar{\gamma}_{S D}$, and $E\left[\gamma_{R_{j} D}\right]=$ $\bar{\gamma}_{R D}$, for any $i=1, \ldots, N_{t}$, any $k=1, \ldots, N_{r r}$, and any $j=1, \ldots, N_{r t}$. The fixed-gain relaying factor is adjusted according to [5] as $G^{2}=E\left[\frac{P_{R}}{P_{S}\left|h_{S_{i} R_{k}}\right|^{2}+N_{0}}\right]$.

\section{B. Antenna Selection Strategy}

The optimal selection criterion chooses the best transmit antennas at $S$ and $R$ that maximize the end-to-end SNR, i.e.,

$$
\left(i^{*}, k^{*}, j^{*}\right)=\arg \max _{i, k, j}\left[\gamma_{i, k, j}\right] .
$$

Note that the optimal scheme entails a large amount of delay and feedback overhead due to the full system CSI required for decision. To alleviate this, relying on the idea pioneeringly proposed in [4], a suboptimal (yet efficient) distributed antenna selection (DAS) scheme is presented next. In this scheme, the local CSI available at $S$ is exploited to its furthest extent in order to assist the decision, incurring a negligible delay and feedback overhead.

The DAS concept is motivated and supported by the following inequality involving the end-to-end SNR:

$$
\gamma_{i, k, j}<\gamma_{S_{i} D}+\gamma_{S_{i} R_{k}} \min \left[\frac{\gamma_{R_{j} D}}{C}, 1\right] \triangleq \tilde{\gamma}_{i} .
$$

The DAS scheme is performed in two time slots [4]. In the first time slot, $D$ sends to $R$ and $S$ a 1-bit reverse pilot signaling. Then, $R$ and $S$ use this bit to estimate their respective local CSIs $\gamma_{R_{j} D}$ and $\gamma_{S_{i} D}$. At this time, based on $\gamma_{R_{j} D}$, the relay selects its optimal antenna to transmit data, by applying the selection rule $j^{*}=\arg \max \left[\gamma_{R_{j} D}\right]$. In the second time slot, $R$ compares its local CSI $\gamma_{R_{j *} D}$ with $C$, yielding two cases:

- Case I: If $\gamma_{R_{j^{*}} D} \geq C, R$ sends to $S$ a 1-bit message "1". In this case, by using (3), $\gamma_{S_{i} D}$ and $\gamma_{S_{i} R_{k}}$, which are available at $S$, are sufficient to apply the selection rule $\max \left\{\tilde{\gamma}_{i, k, j^{*}}\right\}$.

- Case II: If $\gamma_{R_{j^{*}} D}<C, R$ sends to $S$ a 1-bit message " 0 ". In this case, by using (3), the application of $\max \left\{\tilde{\gamma}_{i, k, j^{*}}\right\}$ would require the additional knowledge of $\gamma_{R_{j *} D}$, which is unavailable at $S$. Therefore, somewhat arbitrarily, a suboptimal decision will be employed that depends solely on the maximization of $\gamma_{S_{i} D}$, which is available at $S$. All in all, the TAS rule of the proposed DAS scheme is summarized as follows:

$$
i^{*}= \begin{cases}\bar{i}=\arg \max _{i}\left[\gamma_{S_{i} D}+\max _{k}\left[\gamma_{S_{i} R_{k}}\right]\right], & \text { if } \gamma_{R_{j *} D} \geq C \\ \underline{i}=\arg \max _{i}\left[\gamma_{S_{i} D}\right], & \text { if } \gamma_{R_{j *} D}<C\end{cases}
$$

It is noteworthy that the major advantage of DAS over other antenna selection schemes is its reduced delay/feedback overhead, requiring only a 2-bit pilot/feedback signaling, at the expense of some additional hardware complexity at the source.

\section{PERFormancE ANALYSis}

In this section, the outage probability for the proposed scheme will be analyzed. Such a metric can be defined as the probability that the end-to-end SNR falls below a given threshold, commonly written in terms of a target spectral efficiency $\Re_{0}$, being mathematically formulated as

$$
\begin{aligned}
& P_{\text {out }}^{\text {DAS }}= \\
& \underbrace{\operatorname{Pr}\left(\gamma_{R_{j^{*}} D} \geq C,\left(\gamma_{S_{\bar{i}} D}+\frac{\gamma_{S_{\bar{i}} R_{k^{*}}} \gamma_{R_{j^{*}} D}}{\gamma_{R_{j^{*}} D}+C}\right)<z \triangleq 2^{2 \Re_{0}}-1\right)}_{P_{P_{1}}} \\
& +\underbrace{\operatorname{Pr}\left(\gamma_{R_{j^{*}} D}<C,\left(\gamma_{S_{\underline{i}} D}+\frac{\gamma_{S_{\underline{i}} R_{k^{*}}} \gamma_{R_{j^{*}} D}}{\gamma_{R_{j^{*}} D}+C}\right)<z\right)}_{P_{2}} .
\end{aligned}
$$

Since an exact closed-form expression for the above expression is mathematically intractable, lower and upper bounds for the outage probability will be derived instead, based on the following paramount relationships:

$$
\frac{\gamma_{S_{i} R_{k}}}{2} \min \left[\frac{\gamma_{R_{j} D}}{C}, 1\right] \leq \frac{\gamma_{S_{i} R_{k}} \gamma_{R_{j} D}}{\gamma_{R_{j} D}+C} \leq \gamma_{S_{i} R_{k}} \min \left[\frac{\gamma_{R_{j} D}}{C}, 1\right] .
$$

In order to evaluate the terms $P_{1}$ and $P_{2}$, we first derive a lower bound $P_{1}^{\mathrm{LB}}$ for $P_{1}$, using (6), as follows:

$$
\begin{aligned}
P_{1} & >\operatorname{Pr}\left(\gamma_{R_{j^{*}} D} \geq C,\left(\gamma_{S_{\bar{i}} D}+\gamma_{S_{\bar{i}} R_{k^{*}}} \min \left[\frac{\gamma_{R_{j^{*}} D}}{C}, 1\right]\right)<z\right) \\
& \stackrel{(a)}{=} \operatorname{Pr}\left(\gamma_{R_{j^{*}} D} \geq C, \max _{i}\left(\gamma_{S_{i} D}+\max _{k}\left(\gamma_{S_{i} R_{k}}\right)\right)<z\right) \triangleq P_{1}^{\mathrm{LB}} \\
& =\operatorname{Pr}\left(\gamma_{R_{j^{*}} D} \geq C\right) \operatorname{Pr}\left(\left(\gamma_{S_{i} D}+\max _{k}\left(\gamma_{S_{i} R_{k}}\right)\right)<z\right)^{N_{t}} \\
& =\operatorname{Pr}\left(\gamma_{R_{j^{*}} D} \geq C\right) \\
& \times\left(\int_{0}^{z} f_{\gamma_{S D}}(x) \operatorname{Pr}\left(\left(x+\max _{k}\left(\gamma_{S_{i} R_{k}}\right)\right)<z\right) d x\right)^{N_{t}} \\
& =\operatorname{Pr}\left(\gamma_{R_{j^{*} D}} \geq C\right) \\
& \times(\underbrace{\int_{0}^{z} \frac{1}{\bar{\gamma}_{S D}} e^{-\frac{x}{\gamma_{S D}}}\left(1-e^{-\frac{z-x}{\bar{\gamma}_{S R}}}\right)^{N_{r r}} d x}_{\alpha},
\end{aligned}
$$

where in step (a) we applied the DAS rule given in (4) for $\gamma_{R_{j^{*}} D} \geq C$. The term $\alpha$ can be rewritten using the binomial 
theorem [6, Eq. (1.111)] as

$$
\begin{aligned}
\alpha & =\int_{0}^{z} \frac{1}{\bar{\gamma}_{S D}} e^{-\frac{x}{\bar{\gamma}_{S D}}} \sum_{j=0}^{N_{r r}}\left(\begin{array}{c}
N_{r r} \\
j
\end{array}\right)(-1)^{j} e^{-j \frac{z-x}{\bar{\gamma}_{S R}}} d x \\
& =\sum_{j=0}^{N_{r r}}\left(\begin{array}{c}
N_{r r} \\
j
\end{array}\right)(-1)^{j} e^{-j \frac{z}{\bar{\gamma}_{S R}}} \frac{1}{\bar{\gamma}_{S D}} \int_{0}^{z} e^{j \frac{x}{\bar{\gamma}_{S R}}-\frac{x}{\bar{\gamma}_{S D}}} d x \\
& =\sum_{j=0}^{N_{r r}}\left(\begin{array}{c}
N_{r r} \\
j
\end{array}\right)(-1)^{j} e^{-j \frac{z}{\bar{\gamma}_{S R}}}\left(\frac{\left(1-e^{-\frac{z}{\bar{\gamma}_{S D}}+\frac{j z}{\bar{\gamma}_{S R}}}\right) \bar{\gamma}_{S R}}{j \bar{\gamma}_{S D}-\bar{\gamma}_{S R}}\right) .
\end{aligned}
$$

Then, by substituting (8) in (7), a lower bound for $P_{1}$ can be derived in closed form as

$$
\begin{aligned}
P_{1}^{\mathrm{LB}} & =\left(1-\left(1-e^{-\frac{C}{\bar{\gamma}_{R D}}}\right)^{N_{r t}}\right) \\
& \times\left(\sum_{j=0}^{N_{r r}}\left(\begin{array}{c}
N_{r r} \\
j
\end{array}\right)(-1)^{j}\left(\frac{\left(e^{-j \frac{z}{\bar{\gamma}_{S R}}}-e^{-\frac{z}{\bar{\gamma}_{S D}}}\right) \bar{\gamma}_{S R}}{j \bar{\gamma}_{S D}-\bar{\gamma}_{S R}}\right)\right)^{N_{t}} .
\end{aligned}
$$

In order to assess the asymptotic behavior of $P_{1}^{\mathrm{LB}}$, a high-SNR expression for the term $\alpha$ can be derived from its definition as

$$
\begin{aligned}
\alpha & =\int_{0}^{z} \frac{1}{\bar{\gamma}_{S D}} e^{-\frac{z-y}{\bar{\gamma}_{S D}}}\left(1-e^{-\frac{y}{\bar{\gamma}_{S R}}}\right)^{N_{r r}} d y \\
& \simeq\left(\frac{1}{\bar{\gamma}_{S R}}\right)^{N_{r r}} \frac{1}{\bar{\gamma}_{S D}} \frac{z^{N_{r r}+1}}{\left(N_{r r}+1\right)},
\end{aligned}
$$

which, when replaced in (7), yields a corresponding high-SNR asymptotic lower bound for $P_{1}$ as

$$
P_{1}^{\mathrm{LB}} \simeq\left(1-\left(1-e^{-\frac{C}{\bar{\gamma}_{R D}}}\right)^{N_{r t}}\right)\left(\left(\frac{1}{\bar{\gamma}_{S R}}\right)^{N_{r r}} \frac{1}{\bar{\gamma}_{S D}} \frac{z^{N_{r r}+1}}{\left(N_{r r}+1\right)}\right)^{N_{t}}
$$

Using (6), it can be shown that an upper bound $P_{1}^{\mathrm{UB}}$ for $P_{1}$ and its asymptote can be directly attained by replacing $z$ with $2 z$ into (9) and (11), respectively.

Similarly, a lower bound $P_{2}^{\mathrm{LB}}$ for $P_{2}$ can be formulated as

$$
\begin{aligned}
P_{2} & >\operatorname{Pr}\left(\gamma_{R_{j^{*}} D}<C,\left(\gamma_{S_{\underline{i}} D}+\gamma_{S_{\underline{\underline{i}}} R_{k^{*}}} \min \left[\frac{\gamma_{R_{j^{*}} D}}{C}, 1\right]\right)<z\right) \\
& =\operatorname{Pr}\left(\gamma_{R_{j^{*}} D}<C,\left(\gamma_{S_{\underline{i}} D}+\frac{\gamma_{S_{\underline{\underline{i}}} R_{k^{*}}} \gamma_{R_{j^{*}} D}}{C}\right)<z\right)=P_{2}^{\mathrm{LB}} \\
& =\operatorname{Pr}\left(\gamma_{R_{j^{*}} D}<C,\left(\max _{m}\left[\gamma_{S_{m} D}\right]+\max _{k}\left[\gamma_{S_{i} R_{k}}\right] \frac{\gamma_{R_{j^{*}} D}}{C}\right)<z\right) .
\end{aligned}
$$

By using the principles of probability theory, $P_{2}^{\mathrm{LB}}$ can be elaborated as

$$
\begin{aligned}
& P_{2}^{\mathrm{LB}} \\
& =\int_{0}^{C} f_{\gamma_{R D}}(x) \operatorname{Pr}\left(\left(\max _{m}\left[\gamma_{S_{m} D}\right]+\max _{k}\left[\gamma_{S_{i} R_{k}}\right] \frac{x}{C}\right)<z\right) d x \\
& =\int_{0}^{1} C f_{\gamma_{R D}}(C u) \operatorname{Pr}\left(\left(\max _{m}\left[\gamma_{S_{m} D}\right]+\max _{k}\left[\gamma_{S_{i} R_{k}}\right] u\right)<z\right) d u \\
& =\int_{0}^{1} C f_{\gamma_{R D}}(C u) \int_{0}^{z} f_{\gamma_{S R}}(y) \operatorname{Pr}\left(\left(\max _{m}\left[\gamma_{S_{m} D}\right]+y u\right)<z\right) d y d u \\
& =\int_{0}^{1} C f_{\gamma_{R D}}(C u) \int_{0}^{z} \frac{1}{u} f_{\gamma_{S R}}\left(\frac{v}{u}\right) \operatorname{Pr}\left(\max _{m}\left[\gamma_{S_{m} D}\right]<z-v\right) d v d u \\
& =\int_{0}^{1} \frac{C N_{r t}}{\bar{\gamma}_{R D}} e^{-\frac{C u}{\bar{\gamma}_{R D}}}\left(1-e^{-\frac{C u}{\bar{\gamma}_{R D}}}\right)^{N_{r t}-1} \frac{N_{r r}}{u \bar{\gamma}_{S R}} \\
& \times \underbrace{\int_{0}^{z} e^{-\frac{v}{u \bar{\gamma} S R}}\left(1-e^{-\frac{v}{u \bar{\gamma} S R}}\right)^{N_{r r}-1}\left(1-e^{-\frac{z-v}{\bar{\gamma}_{S D}}}\right)^{N_{t}} d v}_{\varphi} d u
\end{aligned}
$$

where the term $\varphi$ can be calculated using the binomial theorem $[6$, Eq. (1.111)] as

$$
\begin{aligned}
\varphi & =\sum_{m=0}^{N_{t}} \sum_{j=0}^{N_{r r}}\left(\begin{array}{c}
N_{t} \\
m
\end{array}\right)\left(\begin{array}{c}
N_{r r}-1 \\
j
\end{array}\right)(-1)^{m}(-1)^{j} \\
& \times \frac{\left(e^{-\frac{(1+j) z}{u \bar{\gamma}} S R}-e^{-\frac{m z}{\bar{\gamma}_{S D}}}\right) u \bar{\gamma}_{S R} \bar{\gamma}_{S D}}{m u \bar{\gamma}_{S R}-(1+j) \bar{\gamma}_{S D}} .
\end{aligned}
$$

Then, by substituting (14) into (13), a lower bound for $P_{2}$ can be derived in single integral form as

$$
\begin{aligned}
P_{2}^{\mathrm{LB}} & =\int_{0}^{1} \frac{C N_{r t}}{\bar{\gamma}_{R D}} e^{-\frac{C u}{\bar{\gamma}_{R D}}}\left(1-e^{-\frac{C u}{\bar{\gamma}_{R D}}}\right)^{N_{r t}-1} \frac{N_{r r}}{u} \\
& \times \sum_{m=0}^{N_{t}} \sum_{j=0}^{N_{r r}}\left(\begin{array}{c}
N_{t} \\
m
\end{array}\right)\left(\begin{array}{c}
N_{r r}-1 \\
j
\end{array}\right)(-1)^{m}(-1)^{j} \\
& \times \frac{\left(e^{-\frac{(1+j) z}{u \bar{\gamma}_{S R}}}-e^{-\frac{m z}{\bar{\gamma}_{S D}}}\right) u \bar{\gamma}_{S D}}{m u \bar{\gamma}_{S R}-(1+j) \bar{\gamma}_{S D}} d u .
\end{aligned}
$$

In order to assess the asymptotic behavior of $P_{2}^{\mathrm{LB}}$, we simplify the term $\varphi$ defined in (13) by using the binomial theorem [6, Eq. (1.111)] and the MacLaurin series of exponential functions [6, Eq. (1.211.1)], obtaining

$$
\begin{aligned}
\varphi & =\sum_{i=1}^{N_{r r}}\left(\begin{array}{c}
N_{r r}-1 \\
i-1
\end{array}\right)(-1)^{i-1} \int_{0}^{z} e^{-\frac{i v}{u \bar{\gamma}_{S R}}}\left(\frac{z-v}{\bar{\gamma}_{S D}}\right)^{N_{t}} d v \\
& =\sum_{i=1}^{N_{r r}}\left(\begin{array}{c}
N_{r r}-1 \\
i-1
\end{array}\right)(-1)^{i-1} e^{-\frac{i z}{u \bar{\gamma}_{S R}}}\left(\frac{1}{\bar{\gamma}_{S D}}\right)^{N_{t}} \\
& \times \sum_{n=0}^{\infty}\left(\frac{i}{u \bar{\gamma}_{S R}}\right)^{n} \frac{z^{N_{t}+n+1}}{n !\left(N_{t}+n+1\right)} .
\end{aligned}
$$

By substituting (16) into (13) and using again the binomial 
theorem, $P_{2}^{\mathrm{LB}}$ can be rewritten as

$$
\begin{aligned}
P_{2}^{\mathrm{LB}} & =N_{r t} \frac{N_{r r}}{\bar{\gamma}_{S R}}\left(\frac{1}{\bar{\gamma}_{S D}}\right) \sum_{i=1}^{N_{t}}\left(\begin{array}{c}
N_{r r}-1 \\
i-1
\end{array}\right)(-1)^{i-1} \\
& \times \sum_{j=0}^{N_{r t}}\left(\begin{array}{c}
N_{r t}-1 \\
j-1
\end{array}\right)(-1)^{j-1} \sum_{n=0}^{\infty} \\
& \times \underbrace{\frac{z^{N_{t}+n+1} i^{n} \bar{\gamma}_{S R}^{-n}}{n !\left(N_{t}+n+1\right)} \int_{0}^{1} \frac{C}{\bar{\gamma}_{R D}} u^{-n-1} e^{-\frac{i z}{u \bar{\gamma}_{S R}}} e^{-\frac{j C u}{\bar{\gamma}_{R D}}} d u}_{\Phi_{n}} .
\end{aligned}
$$

The behavior of $\Phi_{n}$ in the high-SNR regime is characterized in Apps. A and B. In App. A, $\Phi_{n}$ is determined for $n=0$, yielding

$$
\Phi_{0} \simeq \begin{cases}\frac{z^{N_{t}+1}}{\left(N_{t}+1\right)} \frac{\left(\frac{j C i z}{\bar{\gamma}_{S R} \bar{\gamma}_{R D}}\right)^{n_{1}}}{n_{1} !^{2} \mu_{2}}\left(-\ln \left(\frac{j i z}{\bar{\gamma}_{R D}}\right)\right. & \\ \left.+\psi\left(n_{1}+1\right)-(-1)^{n_{1}} \Gamma\left(-n_{1}, \frac{j C}{\bar{\gamma}_{R D}}\right)\right), & \text { if } N_{r r}=N_{r t} \\ \frac{z^{N_{t}+1}}{\left(N_{t}+1\right)} \frac{\left(\frac{j C i z}{\bar{\gamma}_{S R} \bar{\gamma}_{R D}}\right)^{n_{1}}}{n_{1} !^{2} \mu_{2}} & \\ \left(-\ln \left(\frac{j i z}{\bar{\gamma}_{R D}}\right)-(-1)^{n_{1}} \Gamma\left(-n_{1}, \frac{j C}{\bar{\gamma}_{R D}}\right)\right), & \text { if } N_{r r}<N_{r t} \\ \frac{z^{N_{t}+1}}{\left(N_{t}+1\right)} \frac{\left(\frac{j C i z}{\bar{\gamma}_{S R} \bar{\gamma}_{R D}}\right)^{n_{1}}}{n_{1} !^{2} \mu_{2}}\left(-\ln \left(\frac{j i z}{\bar{\gamma}_{R D}}\right)\right), & \text { if } N_{r r}>N_{r t},\end{cases}
$$

where $\mu_{2} \triangleq \bar{\gamma}_{R D} / \bar{\gamma}_{S R}$ and $n_{1} \triangleq \min \left(N_{r r}, N_{r t}\right)-1$. In App. B, $\Phi_{n}$ is determined for $n>0$, in which two intervals for $n$ are established $\left(1 \leq n \leq n_{1}\right.$ and $\left.n>n_{1}\right)$, yielding

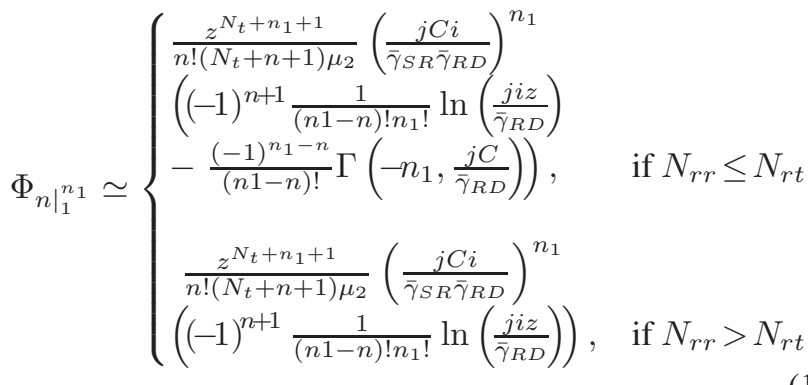

$$
\begin{aligned}
& \Phi_{\left.n\right|_{n_{1}+1} ^{\infty}} \simeq \begin{cases}\frac{z^{N_{t}+n_{1}+1}}{n !\left(N_{t}+n+1\right) \mu_{2}}\left(\frac{j C i}{\bar{\gamma}_{S R} \bar{\gamma}_{R D}}\right)^{n_{1}} & \\
\frac{\left(n-n_{1}-1\right) !}{n_{1} !}(-1)^{n_{1}}, & \text { if } N_{r r}=N_{r t} \\
0, & \text { otherwise. }\end{cases}
\end{aligned}
$$

As done before for the term $P_{1}$, using (6), it can be shown that an upper bound $P_{2}^{\mathrm{UB}}$ for $P_{2}$ and its asymptote can be directly attained by replacing $z$ with $2 z$ into (15) and (17).

Finally, by adding as in (5) the asymptotic bounds derived for $P_{1}$ and $P_{2}$, corresponding asymptotic lower and upper bounds for $P_{\text {out }}$ are obtained, respectively, as

$$
P_{\text {out }}^{\mathrm{DAS}, \mathrm{LB}} \simeq \begin{cases}P_{1}^{\mathrm{LB}}+P_{2}^{\mathrm{LB}}, & \text { if } N_{t}=1 \text { and } N_{r r} \leq N_{r t} \\ P_{2}^{\mathrm{LB}}, & \text { otherwise }\end{cases}
$$

$P_{\text {out }}^{\mathrm{DAS}, \mathrm{UB}} \simeq \begin{cases}P_{1}^{\mathrm{UB}}+P_{2}^{\mathrm{UB}}, & \text { if } N_{t}=1 \text { and } N_{r r} \leq N_{r t} \\ P_{2}^{\mathrm{UB}}, & \text { otherwise. }\end{cases}$

\section{Remarks:}

1. Using (21), (22), and the asymptotic bounds for $P_{1}$ and $P_{2}$, it can be shown that the proposed DAS scheme achieves full diversity order, being equal to $N_{t}+\min \left(N_{r r}, N_{r t}\right)$.

2. As shown in (17), both lower and upper asymptotic bounds for $P_{2}$ are given in terms of an infinite series. In order to observe the convergence of this series, two cases are analyzed: (i) when $N_{r r} \neq N_{r t}$, only the first $n_{1}+1$ terms are taken into consideration, since the series is zero for terms greater than $n_{1}$; (ii) when $N_{r r}=N_{r t}$, similarly to [4], the convergence is proved using the convergence test of $[6, \mathrm{Eq}$. $(0.223)]$.

\section{NumERICAL RESUlts AND Discussions}

In this section, representative numerical results are presented and Monte Carlo simulations are run to support the derived analytical bounds. In our plots, as a sample case, we assume that the target spectral efficiency is $\Re_{0}=1 \mathrm{bit} / \mathrm{s} / \mathrm{Hz}$ and that the path loss exponent is $\beta=4$. We also assume that the channel mean power is proportional to $d^{-\beta}$, with $d$ being the distance between the transceivers. The distance between $S$ and $D$ is normalized to unity, as in $[4]^{2}$. Figs. 1 and 2 show the outage probability for two major system configurations, namely $\left\{N_{t}=2, d_{S R}=0.7\right\}$ and $\left\{N_{t}=3, d_{S R}=0.8\right\}$, respectively. It is noteworthy that, in each case, the relay has been placed at the position $d_{S R}$ that provides the best outage performance, and such a position was previously established through simulations. These prior simulation results have not been presented here due to space limitations. From the figures, note that the performed analysis for the exact and asymptotic bounds is validated, and that the diversity order, determined as $N_{t}+\min \left(N_{r r}, N_{r t}\right)$, is verified. For instance, using the system configuration $\left\{N_{t}=2, N_{r r}=2, N_{r t}=2\right\}$ the diversity order equals 4, which agrees with the asymptotes's slope in Fig. 2. In addition, both figures compares the performance of the proposed DAS and optimal TAS schemes, which can be seen to be very similar.

\section{APPENDiX A}

HIGH-SNR EXPRESSION FOR THE TERM $\Phi_{0}$ IN (17)

Here we consider $\Phi_{n}$ for $n=0$, which is defined in (17) as

$$
\Phi_{0}=\frac{z^{N_{t}+1}}{\left(N_{t}+1\right)} \frac{C}{\bar{\gamma}_{R D}} \underbrace{\int_{0}^{1} u^{-1} e^{-\frac{i z}{u \bar{\gamma} S R}} e^{-\frac{j C u}{\bar{\gamma}_{R D}}} d u}_{\rho_{0}} .
$$

The term $\rho_{0}$ can be rewritten through the help of $[6$, Eq. (3.471.9)], [6, Eq. (1.211.1)], and [6, Eq. (8.446)] as (24), given at the top of the next page, in which $K_{n}(\cdot)$ denotes

\footnotetext{
${ }^{2}$ Again, as in [4], we assume a linear network topology, in which $S$ and $R$ transmit with the same SNR $P$, and $d_{S D}=d_{S R}+d_{R D}$, where $d_{S D}$, $d_{S R}$, and $d_{R D}$ represent the distance of the links $S \rightarrow D, S \rightarrow R$, and $R \rightarrow D$, respectively. The corresponding average link SNRs can be formulated as $\bar{\gamma}_{S D}=P d_{S D}^{-\beta}, \bar{\gamma}_{S R}=P d_{S R}^{-\beta}$, and $\bar{\gamma}_{R D}=P d_{R D}^{-\beta}$.
} 


$$
\begin{aligned}
\rho_{0} & =2 K_{0}\left(2 \sqrt{\frac{j C i z}{\bar{\gamma}_{S R} \bar{\gamma}_{R D}}}\right)-\int_{1}^{\infty} u^{-1} e^{-\frac{j C u}{\bar{\gamma}_{R D}}} \sum_{l=0}^{\infty} \frac{\left(-\frac{i z}{u \overline{\bar{\gamma}_{S R}}}\right)^{l}}{l !} d u=-\ln \frac{j C i z}{\bar{\gamma}_{S R} \bar{\gamma}_{R D}} \sum_{k_{1}=0}^{\infty} \frac{\left(\sqrt{\frac{j C i z}{\bar{\gamma}_{S R} \bar{\gamma}_{R D}}}\right)^{2 k_{1}}}{k_{1} !^{2}}+\sum_{k_{2}=0}^{\infty} \frac{\left(\sqrt{\frac{j C i z}{\bar{\gamma}_{S R} \bar{\gamma}_{R D}}}\right)^{2 k_{2}}}{k_{2} !^{2}} \\
& \times 2 \psi\left(k_{2}+1\right)-\sum_{l=0}^{\infty} \frac{\left(-\frac{i z}{\bar{\gamma}_{S R}}\right)^{l}}{l !}\left(\frac{j C}{\bar{\gamma}_{R D}}\right)^{l} \Gamma\left(-l, \frac{j C}{\bar{\gamma}_{R D}}\right) .
\end{aligned}
$$

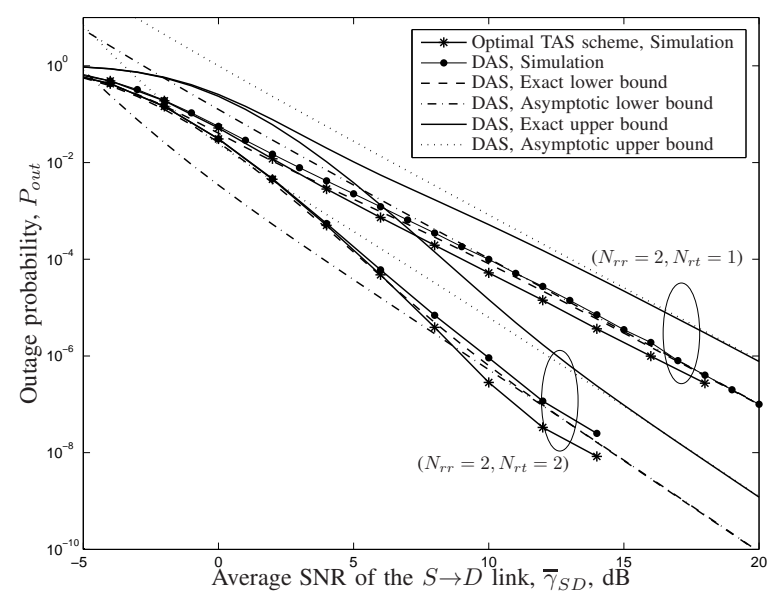

Fig. 1. Outage probability versus average SNR of the $S \rightarrow D$ link for different AS schemes $\left(d_{S R}=0.7, N_{t}=2\right)$.

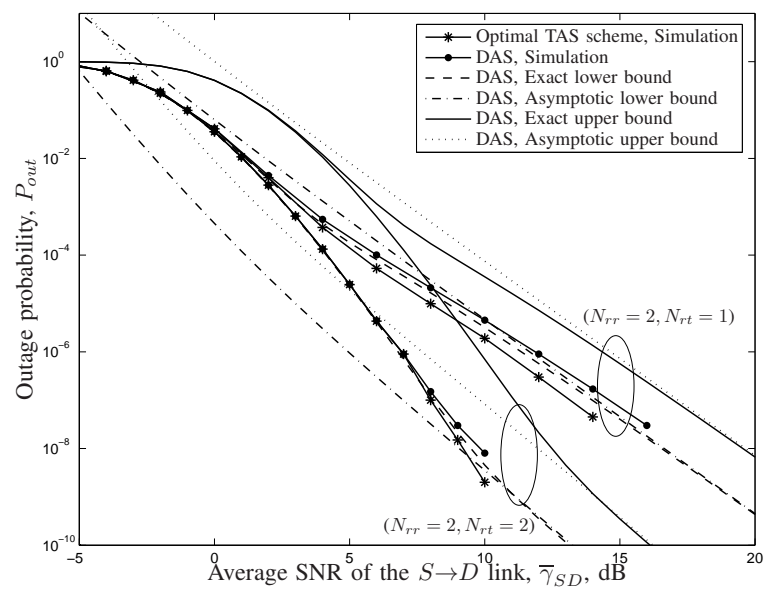

Fig. 2. Outage probability versus average SNR of the $S \rightarrow D$ link for different AS schemes $\left(d_{S R}=0.8, N_{t}=3\right)$.

the $n$ th-order modified Bessel function of the second kind [6, Eq.(8.446)], $\psi(\cdot)$ represents the Euler psi function [6, Eq. (8.360)] and $\Gamma(\cdot)$ is the gamma function [6, Eq. (8.310.1)]. It is important to mention that in order to conduct the high-SNR analysis of the inner expression that defines $\Phi_{0}$ in (17), the outer summations in $i$ and $j$ must be considered. To this end, we define the variable $\lambda_{0}$ as

$$
\lambda_{0}=\sum_{i=1}^{N_{r r}}\left(\begin{array}{c}
N_{r r}-1 \\
i-1
\end{array}\right)(-1)^{i-1} \sum_{j=1}^{N_{r t}}\left(\begin{array}{c}
N_{r t}-1 \\
j-1
\end{array}\right) \times(-1)^{j-1} \Phi_{0} .
$$

Plugging (24) into (23) and this into (25), $\lambda_{0}$ can be rewritten as

$$
\begin{aligned}
& \lambda_{0}=\frac{z^{N_{t}+1}}{\left(N_{t}+1\right)} \frac{C}{\bar{\gamma}_{R D}}\left(-\sum_{k_{1}=0}^{\infty} \frac{\left(\frac{C z}{\bar{\gamma}_{S R} \bar{\gamma}_{R D}}\right)^{k_{1}}}{k_{1} !^{2}} \sum_{i=1}^{N_{r r}}\left(\begin{array}{c}
N_{r r}-1 \\
i-1
\end{array}\right) i^{k_{1}}\right. \\
& \times(-1)^{i-1} \sum_{j=1}^{N_{r t}}\left(\begin{array}{c}
N_{r t}-1 \\
j-1
\end{array}\right)(-1)^{j-1} j^{k_{1}}\left(\ln \left(\frac{j}{\bar{\gamma}_{R D}}\right)+\ln (i z)\right) \\
& +\sum_{k_{2}=0}^{\infty} \frac{\left(\frac{C z}{\bar{\gamma}_{S R} \bar{\gamma}_{R D}}\right)^{k_{2}}}{k_{2} !^{2}} \psi\left(k_{2}+1\right) \sum_{i=1}^{N_{r r}}\left(\begin{array}{c}
N_{r r}-1 \\
i-1
\end{array}\right)(-1)^{i-1} i^{k_{2}} \\
& \times \sum_{j=1}^{N_{r t}}\left(\begin{array}{c}
N_{r t}-1 \\
j-1
\end{array}\right)(-1)^{j-1} j^{k_{2}}-\sum_{l=0}^{\infty} \frac{\left(\frac{-C z}{\bar{\gamma}_{S R} \bar{\gamma}_{R D}}\right)^{l}}{l !^{2}} \sum_{i=1}^{N_{r r}}\left(\begin{array}{c}
N_{r r}-1 \\
i-1
\end{array}\right) \\
& \left.\times(-1)^{i-1} i^{l} \sum_{j=1}^{N_{r t}}\left(\begin{array}{c}
N_{r t}-1 \\
j-1
\end{array}\right)(-1)^{j-1} j^{l} \Gamma\left(-l, \frac{j C}{\bar{\gamma}_{R D}}\right)\right) .
\end{aligned}
$$

Observe that $\lambda_{0}$ is composed by the sum of three terms, corresponding to the summations in $k_{1}, k_{2}$, and $l$. Using the fact that $\sum_{a=1}^{b}\left(\begin{array}{l}b-1 \\ a-1\end{array}\right)(-1)^{a-1} a^{c}$ is null for $c=0,1,2, \ldots, b-2$, after a careful inspection it was attested that each term presents a different behavior at high-SNR regime, and this will be described next. The first term is non-null for values of $k_{1}$ greater than $\min \left(N_{r r}, N_{r t}\right)-1$, the second term is non-null for values of $k_{2}$ greater than $\max \left(N_{r r}, N_{r t}\right)-1$, and the third term is non-null for values of $l$ greater than $N_{r r}-1$. Using this and preserving only the lowest-order terms so as to derive a high-SNR expression, we arrive at three cases: (i) if $N_{r r}=N_{r t}$, the three terms must be considered; (ii) if $N_{r r}<N_{r t}$, the first and third terms must be considered; and (iii) if $N_{r r}>N_{r t}$, only the first term must be considered. In all the cases, the diversity order of $\lambda_{0}$ is observed to be given by $n_{1} \triangleq \min \left(N_{r r}, N_{r t}\right)-1$. By incorporating these results into the definition of $\Phi_{0}$ given by (23) and (24), we finally arrive at the high-SNR expression for $\Phi_{0}$ as given in (18).

\section{APPENDIX B}




$$
\begin{aligned}
& \rho_{n} \stackrel{(b)}{=} 2 \bar{\gamma}_{S R}^{-\frac{n}{2}}\left(\frac{i z \bar{\gamma}_{R D}}{j C}\right)^{-\frac{n}{2}} K_{n}\left(2 \sqrt{\frac{j C i z}{\bar{\gamma}_{S R} \bar{\gamma}_{R D}}}\right)-\sum_{l=0}^{\infty} \frac{\bar{\gamma}_{S R}^{-n}\left(-\frac{i z}{\bar{\gamma}_{S R}}\right)^{l}}{l !} \int_{1}^{\infty} u^{-n-l-1} e^{-\frac{j C u}{\bar{\gamma}_{R D}}} d u \\
& \stackrel{(c)}{=} 2 \bar{\gamma}_{S R}^{-\frac{n}{2}}\left(\frac{i z \bar{\gamma}_{R D}}{j C}\right)^{\frac{-n}{2}} \sum_{m_{1}=0}^{n-1} \frac{(-1)^{m_{1}}\left(n-m_{1}-1\right)}{m_{1} !}\left(\frac{j C i z}{\bar{\gamma}_{S R} \bar{\gamma}_{R D}}\right)^{m_{1}-\frac{n}{2}}+2 \bar{\gamma}_{S R}^{-n}\left(\frac{i z \bar{\gamma}_{R D}}{j C}\right)^{-\frac{n}{2}}(-1)^{n+1}
\end{aligned}
$$

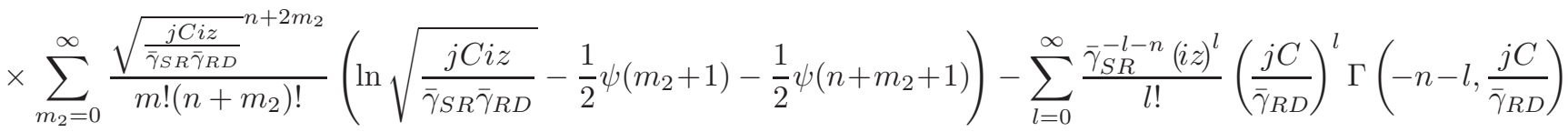

$$
\begin{aligned}
& =\sum_{m_{1}=0}^{n-1}(-1)^{m_{1}} \frac{\left(n-m_{1}-1\right)}{m_{1} !} z^{m_{1}-n} \bar{\gamma}_{S R}^{-m_{1}} i^{m_{1}}\left(\frac{j C}{\bar{\gamma}_{R D}}\right)^{m_{1}}+2 \sum_{m_{2}=0}^{\infty} \frac{1}{m_{2} !\left(n+m_{2}\right) !} z^{m_{2}}\left(\frac{j C}{\bar{\gamma}_{R D}}\right)^{m_{2}+n}\left(\frac{i}{\bar{\gamma}_{S R}}\right)^{n+m_{2}} \\
& \times\left(\ln \sqrt{\frac{j C i z}{\bar{\gamma}_{S R} \bar{\gamma}_{R D}}}-\frac{1}{2} \psi\left(m_{2}+1\right)-\frac{1}{2} \psi\left(n+m_{2}+1\right)\right)-\sum_{l=0}^{\infty} \frac{\bar{\gamma}_{S R}^{-l-n} i^{n+l} z^{l}}{l !}\left(\frac{j C}{\bar{\gamma}_{R D}}\right)^{l+n} \Gamma\left(-n-l, \frac{j C}{\bar{\gamma}_{R D}}\right) . \\
& \lambda_{n}=\frac{z^{N_{t}+n+1}}{n !\left(N_{t}+n+1\right)} \frac{C}{\bar{\gamma}_{R D}}\left(\sum_{m_{1}=0}^{n-1}(-1)^{m_{1}} \frac{\left(n-m_{1}-1\right)}{m_{1} !} \bar{\gamma}_{S R}^{-m_{1}} z^{m_{1}}\left(\frac{C}{\bar{\gamma}_{R D}}\right)^{m_{1}} \sum_{j=1}^{N_{r t}}\left(\begin{array}{c}
N_{r t}-1 \\
j-1
\end{array}\right)(-1)^{j-1} j^{m_{1}} \sum_{i=1}^{N_{r r}}\left(\begin{array}{c}
N_{r r}-1 \\
i-1
\end{array}\right)\right.
\end{aligned}
$$

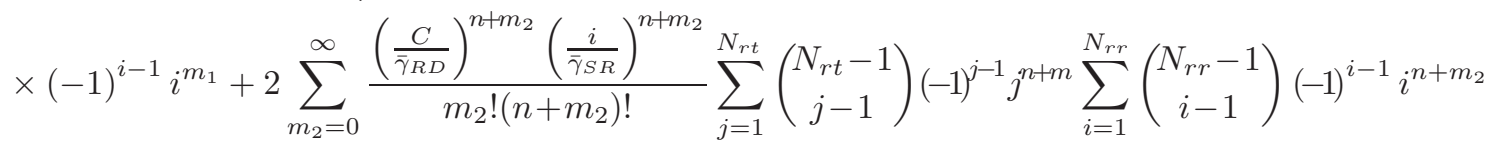

$$
\begin{aligned}
& \times\left(\ln \sqrt{\frac{j C i z}{\bar{\gamma}_{S R} \bar{\gamma}_{R D}}}-\frac{1}{2} \psi\left(m_{2}+1\right)-\frac{1}{2} \psi\left(n+m_{2}+1\right)\right)-\sum_{l=0}^{\infty} \frac{\bar{\gamma}_{S R}^{-l-n}(-z)^{l}}{l !}\left(\frac{C}{\bar{\gamma}_{R D}}\right)^{l} \sum_{j=1}^{N_{r t}}\left(\begin{array}{c}
N_{r t}-1 \\
j-1
\end{array}\right)(-1)^{j-1} \\
& \left.\times j^{n+l} \sum_{i=1}^{N_{r r}}\left(\begin{array}{c}
N_{r r}-1 \\
i-1
\end{array}\right)(-1)^{i-1} i^{n+l} \Gamma\left(-n-l, \frac{j C}{\bar{\gamma}_{R D}}\right)\right) .
\end{aligned}
$$

HIGH-SNR EXPRESSION FOR THE TERM $\Phi_{n}$ IN (17), $n>0$

We now consider $\Phi_{n}$ for $n>0$, which is defined in (17) as

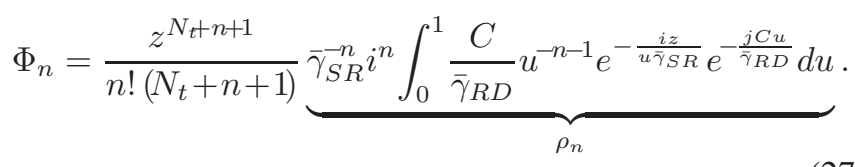

The term $\rho_{n}$ can be rewritten as (28), given at the top of this page, in which we have used [6, Eq. (3.471.9)] and [6, Eqs. (8.446) and (3.381.3)] in steps (c) and (d), respectively. The analysis that follows is similar to that in App. A. To this end, we define the variable $\lambda_{n}$ as

$$
\lambda_{n}=\sum_{i=1}^{N_{r r}}\left(\begin{array}{c}
N_{r r}-1 \\
i-1
\end{array}\right)(-1)^{i-1} \sum_{j=1}^{N_{r t}}\left(\begin{array}{c}
N_{r t}-1 \\
j-1
\end{array}\right)(-1)^{j-1} \Phi_{n} .
$$

Plugging (28) into (27) and this into (29), $\lambda_{n}$ can be rewritten as (30), given at the top of this page. Observe that, similarly to $\lambda_{0}, \lambda_{n}$ is composed by the sum of three terms, corresponding to the summations in $m_{1}, m_{2}$, and $l$. The first term is non-null for values of $m_{1}$ greater than $\max \left(N_{r r}, N_{r t}\right)-1$; the second term is non-null for values of $m_{2}$ greater than $\min \left(N_{r r}, N_{r t}\right)-1$; and the third term is non-null for values of $l$ greater than $N_{r r}-1$. Using this and preserving only the lowest-order terms so as to derive a high-SNR expression, we arrive at three cases: (a) if $N_{r r}=N_{r t}$, the three terms must be considered; (b) if $N_{r r}<N_{r t}$, the second and third terms must be considered; and (c) if $N_{r r}>N_{r t}$, only the second term must be considered. In all the cases, the diversity order of $\lambda_{n}$ is observed to be given by $n_{1} \triangleq \min \left(N_{r r}, N_{r t}\right)-1$. By incorporating these results into the definition of $\Phi_{n}$ given by (27) and (28), we finally arrive at the high-SNR expression for $\Phi_{n}$ as given in (19) and (20).

\section{REFERENCES}

[1] S. Sanayei and A. Nosratinia, "Antenna selection in MIMO systems," IEEE Commun. Mag., vol. 42, no. 10, pp. 68-73, Oct. 2004.

[2] S. Peters and R. Heath, "Nonregenerative MIMO relaying with optimal transmit antenna selection," IEEE Signal Process. Lett, vol. 15, pp. 421424, 2008.

[3] G. Amarasuriya, C. Tellambura, and M. Ardakani, "Performance analysis framework for transmit antenna selection strategies of cooperative MIMO AF relay networks," IEEE Trans. Veh. Technol., vol. 60, no. 7, pp. 30303044, Sep. 2011.

[4] H. Ding, J. Ge, D. da Costa, and T. Tsiftsis, "A novel distributed antenna selection scheme for fixed-gain amplify-and-forward relaying systems," IEEE Trans. Veh. Technol., vol. 61, no. 6, pp. 2836-2842, Jul. 2012.

[5] G. Karagiannidis, "Performance bounds of multihop wireless communications with blind relays over generalized fading channels," IEEE Trans. Wireless Commun., vol. 5, no. 3, pp. 498-503, Mar. 2006.

[6] I. S. Gradshteyn and I. M. Ryzhik, Table of Integrals, Series, and Products, 7th ed. San Diego, CA: Academic Press, 2007. 